\title{
Cryptographic Scheme for Lightweight Device Such as IoT using Pseudo Stream Cipher and Trigonometric Technique with Key Generation
}

\author{
Bhaskar Prakash Kosta, Pasala Sanyasi Naidu
}

\begin{abstract}
The Internet of Things (IoT) being a promising innovation of things to come and is required to associate billions of gadgets. Web of things (IoT) gadgets have been generally utilized, and Electronic correspondence is expanded quickly. The expanded number of correspondence is required to create piles of information and the security of information can be a danger. Information gathered by the IoT gadgets and the information which IoT gadget send might be the portal for an assailant to break client security. To guarantee secure correspondence between IoT centers and central point(server), a cryptographic plan for lightweight gadgets is proposed. In this plan, we make utilize pseudo stream cipher with key generation for rearranging key synchronization and improving security.. The common verification, secret key for meeting synchronization and refreshing secret key for session are finished by trading scrambled messages. Likewise, the key length and update cycle for mystery key for meeting are adaptable as indicated by application. Keys are created from mystery key for meeting for improving the security. We contrasted the plan's security and execution and some lightweight plans. As indicated by the investigation, the proposed plan can give greater security includes low overhead of correspondence which is correct for IoT Node with restricted resource and power. Encryption and decoding is finished utilizing trigonometric ideas and by utilizing the idea of stream figure. Trigonometric ideas are lightweight and improve the security up by an extraordinary degree by diminishing the odds of cryptanalysis. When contrasted with different calculations like Hill figure, RC4, RSA and Present(Lightweight square figure) and so forth, the proposed calculation gives better execution
\end{abstract}

Keywords: lightweight cryptographic scheme; internet of things; pseudo stream cipher.

\section{INTRODUCTION}

Colossal collection of IoT devices is there and they have some portion of usage in step by step life, industry creation which pulled in various researchers to do their assessment in the field of IoT. IoT is a model that consolidates regular elements with the ability to distinguish and talk with singular contraptions using Internet [1]. An IoT device does various

Revised Manuscript Received on May 15, 2020.

* Correspondence Author

Bhaskar Prakash Kosta*, Research Scholar, Department of CSE, GITAM Institute of Technology, GITAM Deemed to be University, Vishakapatnam, AP, India.

Dr. Pasala Sanyasi Naidu, Assoc Professor, Department of CSE, GITAM Institute of Technology, GITAM Deemed to be University, Vishakapatnam, AP, India

(C) The Authors. Published by Blue Eyes Intelligence Engineering and Sciences Publication (BEIESP). This is an open access article under the CC BY-NC-ND license (http://creativecommons.org/licenses/by-nc-nd/4.0/) things anyway when the data moves or stays in center points (IoT or other center) its security is standing up to extraordinary challenges [2]. Those IoT center points which have compelled figuring resources and low power for data which it needs to transmit is issue and a pariah can without a very remarkable stretch break the security as it needs effective affirmation [3][4]. IoT devices are mind boggling , as they can be moved nearer from wherever on the planet. Generally IoT devices are used to assemble data for example, the temperature sensors will record the current condition temperature or inner warmth level. It is seen that various undertakings have shown a creating excitement towards use of IoT. Various employments of IoT in social protection endeavors are discussed in and the improvement openings in human administrations got by IoT will be enormous. A couple of data which the IoT contraption assemble ex heartbeat may require assurance, in like manner this data may incite the encroachment of customer security as the security levels are low. Thought there are various cryptography calculation yet they are not sensible for IoT center points because of advantage and power hindrance in like manner many light weight calculation are open yet they require identical estimation limit on the different sides of correspondence. For some IoT contraptions essentialness and figuring resources are the requirement and cutting down this overhead is critical. Stream figure use XOR action to perform encryption and deciphering, give better correspondence security yet complex key organization gives even more overhead (continuously number of cycle) We propose a lightweight cryptographic figuring using pseudo stream figure and trigonometric limit with key age, which performs shared affirmation , secret key for meeting synchronization and data encryption and disentangling using trigonometric limit with most outrageous security and least overhead of center points . This arrangement consolidates three phases for instance shared affirmation stage, meeting key synchronization and data encryption stage and meeting key update stage. Here riddle key for meeting is delivered by server(so that the load on IoT focus can be reduced) and synchronization of the key is done by three encoded correspondence. Using this riddle key for meeting , both server and focus will make key for encryption or decryption(using four phases portrayed later). Further for regular approval, meeting key synchronization and data encryption and for meeting key update here two level key structures is used which is made out of a fixed private key and a ground-breaking meeting key.

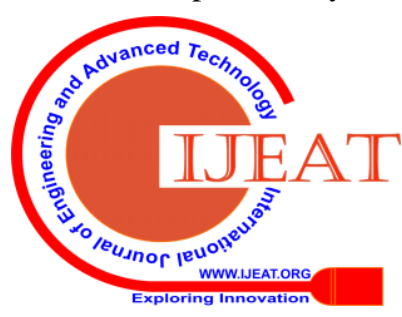


The mystery key is organized prevalently for confirming hotspot for authority and gatherer for source. It is taken care of locally on both server and center point.

Puzzle key for meeting key are made for data encryption and unraveling which changes infrequently and using this key encryption key and unscrambling key are created and used by trigonometric limit (another numerical procedure to prevent cryptanalysis)[19] for encryption and unscrambling. The riddle key gathering length and the update meeting key period can move as showed by the need of express application. The arrangement is versatile and suitable for IoT device security confirmation.

\section{AUTHENTICATION (LIGHTWEIGHT) AND CRYPTOGRAPHIC WAYS}

To offer security to the data which IoT needs to transmit various lightweight approval and cryptographic plans are proposed. The necessity for the lightweight cryptography have been comprehensively discussed [5],[6] also the insufficiencies of the IoT with respect to constrained contraptions are included

RFID has been extensively used in the field of modified ID as a result of its bit of room, for instance, non-contact, convenience, speed and immovable quality anyway its data or information that is moved is unprotected against different ambush. To offer security to the data transmitted by RIFD, Chien (2007) went with ultra-lightweight show called SASI [7]. The show uses XOR, round move and other action to perform shared approval. Regardless, the writing [8] pointed out that the show has an issue, for instance, label following and key spillage. In 2009, another ultra-lightweight show Gossamer was proposed [9]. In 2012, Tina put forth a light weight show using change action to scatter the solicitation for bits [10]. In any case, they notwithstanding everything can't maintain a strategic distance from replay, non synchronization or a couple of ambushes [11]. In 2018, Yuxin and Wang put forth a light weight show using dynamic key[12], this figuring endeavored to take out a part of the above issues, in any case, it moreover has an issue with key i.e examination of data transmitted should be conceivable if the update time period for secret key for meeting is colossal.

To offer security to the data moving in remote sensor organize there are various lightweight arrangement, for instance, Present-square cipher(2007)[13] and Simon and Speck-square cipher(2013) [14]. In any case, as per my finding it has package of estimation unpredictability which may not proper for low resource contraption, for instance, IoT.

Under the condition, we present a lightweight cryptographic arrangement. With the help of clear trigonometric action, encryption and disentangling are done which diminishes preparing usage and security is extended by delivering the key from the got key. This created key forms the security as the outcast won't be in position to do any assessment to calculate the key and a portion of data transmitted can't be recouped. In this arrangement another framework is used to perform approval and meeting key synchronization is done by creating two mixed messages. In this arrangement the server makes the key and passes it to focus point or center point by scrambling it, this lessens the overhead of center. In like manner this arrangement can hinder ambush effectively because of strong confirmation and respectability on the data.

\section{PROPOSED SCHEME DESCRIPTION}

In this area, we will depict the lightweight cryptographic plan utilizing pseudo stream and trigonometric with key generation in detail. The proposed conspire has three stages as follows:

1) Mutual confirmation stage.

2) Secret key for Session synchronization and information encryption stage.

3) Session key update stage.

Prior to portrayal, we right off the bat sum up the documentations utilized all through this paper in Table I and Table II.

Notations Table I

\begin{tabular}{|c|c|}
\hline Symbol & Description \\
\hline $\mathrm{CKi}$ & $\begin{array}{l}\text { A secure pre-shared key between } \\
\text { the center and the switch or server }\end{array}$ \\
\hline IDi & The identity of hub i \\
\hline AIDi & The alias of entity i \\
\hline di & Function generation \\
\hline SKSi & $\begin{array}{c}\text { The mystery key produced by the } \\
\text { server }\end{array}$ \\
\hline $\mathrm{Ri}$ & $\begin{array}{l}\text { A irregular number generated by a } \\
\text { Pseudorandom Number Generator } \\
\text { (PRNG) }\end{array}$ \\
\hline h $()$. & A single direction hash work \\
\hline & OR operator \\
\hline$\oplus$ & XOR activity \\
\hline
\end{tabular}

Table II. Notations in this paper.

Symbol Definition

\begin{tabular}{ll}
\hline Node $_{a}$ & An IoT hub named Node \\
M1N & M1 value generated by Node \\
AIDN & The Alias ID generated by Node \\
M2N & M2 value generated by Node \\
M11N & M11 value generated by Node \\
M22N & M22 value generated by Node \\
a
\end{tabular}

R1N The first Random value(R1) generated by Node

R1SNV The server version of Random1(R1N) generated by Node

AIDSNV The server version of AID(AIDN) generated by Node

M2S M2 value generated by Server

AIDS The Alias ID generated by Server

M11S M11 value generated by Server

M22S M22 value generated by Server

R2S The second Random value(R2S) generated by Server

R2NSV The Node version of Random2(R2S)

server

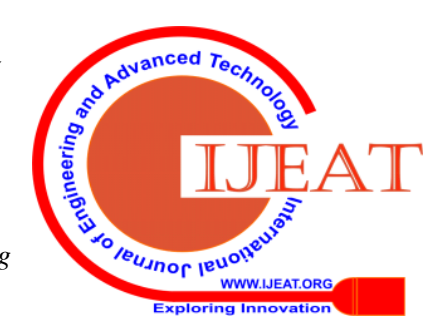




\begin{tabular}{|c|c|}
\hline KS & The secret key for session \\
\hline SKS & $\begin{array}{l}\text { Encrypted secret key for session with } \\
\mathrm{CK}_{\mathrm{a}}\end{array}$ \\
\hline CKS & $\begin{array}{l}\text { Check value generated by server to be } \\
\text { checked by Node }{ }_{a} \text { for getting an assurance } \\
\text { the secret key for session is coming from } \\
\text { correct server. }\end{array}$ \\
\hline ICKN & $\begin{array}{l}\text { Check value generated by } \text { Node }_{a} \text { to } \\
\text { check with MCKS }\end{array}$ \\
\hline HECKN & $\begin{array}{l}\text { Check value generated by Node }{ }_{a} \text { to be } \\
\text { checked by server for getting an } \\
\text { assurance the secret key for session is } \\
\text { received by correct Node. }\end{array}$ \\
\hline HECKS & $\begin{array}{l}\text { Check value generated by server to check } \\
\text { with CHECKN. }\end{array}$ \\
\hline AS & $\begin{array}{l}\text { Final Assurance value generated by } \\
\text { server to be checked by Node }{ }_{a} \text { for } \\
\text { getting an assurance that his previous } \\
\text { reply received by correct server. }\end{array}$ \\
\hline $\mathrm{AN}$ & $\begin{array}{l}\text { Final Assurance value generated by } \text { Node }_{a} \text { to } \\
\text { check with FAS }\end{array}$ \\
\hline IUSKS & $\begin{array}{l}\text { Message for change secret key for } \\
\text { upcomming session }\end{array}$ \\
\hline IUSKS & $\begin{array}{l}\text { Encrypted information for change } \\
\text { secret key for upcomming session } \\
\text { with meeting key }\end{array}$ \\
\hline & Data in plain text \\
\hline & Analysing data for data acceptance \\
\hline
\end{tabular}

\section{A. Mutual Conformation Stage:}

Fixed security key is used in this arrangement for affirmation. Expecting that each middle point has a unique ID and a contrasting pre-shared key. The pre-shared key of center point is simply taken care of locally to ensure its security. The inside point stores the key and its own ID. The server stores the looking at once-over of the center point ID and the affirmation key. ID looks at to the key independently. For example, Node $_{\mathrm{a}}$ has its exceptional IDi and a looking at arrangement key CKa. In this stage, Node $_{a}$ sends its IDi to the server directly off the bat. The common affirmation stage is showed up in Figure1 and the methods are portrayed as follows:

Stage 1: Node $_{\mathrm{a}} \rightarrow$ Server: $\{$ IDi $\}$. The Node ${ }_{\mathrm{a}}$ presents its own IDi to the central point.

Stage 2: Server $\rightarrow$ Node $_{\mathrm{a}}:\left\{\int 2, \int 3\right\}$. Subsequent to getting the message, server discovers CKi as indicated by the IDi. At that point server creates work $\int 1, \int 2, \int 3$ :

$$
\begin{aligned}
& \int 1=\mathrm{h}(\mathrm{IDi} \mid \mathrm{CKi}) \\
& \int 2=\mathrm{h}\left(\int 1\right) \\
& \int 2=\mathrm{CKi} \oplus \int 1
\end{aligned}
$$

Then, server sends $\int 2, \int 3$ to Node $_{\mathrm{a}}$.

Stage 3: Node $_{\mathrm{a}} \rightarrow$ Server: $\{\mathrm{M} 1 \mathrm{~N}, \mathrm{M} 2 \mathrm{~N}\}$. After receiving the message, Node $_{a}$ generates Random ${ }_{1}$ and computes M1N,M2N and AIDN using the IDi as follows:

$$
\begin{gathered}
\mathrm{M} 1 \mathrm{~N}=\mathrm{h}\left(\int 2\right) \oplus \text { Random }_{1} \\
\mathrm{AIDN}=\mathrm{h}\left(\text { Random }_{1}\right) \oplus \mathrm{IDi} \\
\mathrm{M} 2 \mathrm{~N}=\mathrm{h}\left(\text { Random }_{1}|\mathrm{M} 1 \mathrm{~N}| \text { AIDN }\right)
\end{gathered}
$$

Then, the Node $a$ sends M1N , M2N to server.
Stage 4: Server $\rightarrow$ Node $_{\mathrm{a}}:\{$ AIDS , M22S $\}$. After receiving the message, server computes:

$$
\begin{aligned}
& \mathrm{R} 1 \mathrm{SNV}=\mathrm{M} 1 \mathrm{~N} \oplus \mathrm{h}\left(\int_{2}\right) \\
& \text { AIDSNV=h(R1SNV) } \oplus \mathrm{IDi} \\
& \mathrm{M} 2 \mathrm{~S}=\mathrm{h}(\mathrm{R} 1 \mathrm{SNV}|\mathrm{M} 1 \mathrm{~N}| \text { AIDSNV })
\end{aligned}
$$

If M2S=M2N, server considers Node $_{a}$ has a legitimate personality and creates data for self validation by Node ${ }_{a}$ i.e it generates Random 2 (R2S) and computes AIDS, M11S, SKS (Secret Key created by server) and M22S as follows:

$$
\begin{aligned}
& \text { AIDS }=\text { R2S } \oplus \mathrm{h}(\mathrm{IDi}) \\
& \mathrm{M} 11 \mathrm{~S}=\int 1 \oplus \mathrm{h}(\mathrm{IDi}) \\
& \mathrm{M} 22 \mathrm{~S}=\mathrm{h}(\mathrm{M} 11 \mathrm{~S}|\mathrm{AIDS}| \mathrm{R} 2 \mathrm{~S})
\end{aligned}
$$

Then server sends AIDS, M22S to Node . Something else, central point removes the correspondence.

Stage 5: Node $_{a}$ gets AIDS and M22S and computes MCK as follows:

$$
\begin{aligned}
& \text { R2NSV=AIDS } \oplus \mathrm{h}(\mathrm{IDi}) \\
& \int 1=\int 3 \oplus \mathrm{CKi} \\
& \mathrm{M} 11 \mathrm{~N}=\int 1 \oplus \mathrm{h}(\mathrm{IDi}) \\
& \mathrm{M} 22 \mathrm{~N}=\mathrm{h}(\mathrm{M} 11 \mathrm{~N} \mid \text { AIDS } \mid \text { R2NSV })
\end{aligned}
$$

If M22N=M22S, Node $_{a}$ considers central point personality is legitimate and keep correspondence. Something else, Node removes the correspondence.-

The Node $\underline{a}_{\underline{a}}$

The Server

IDi

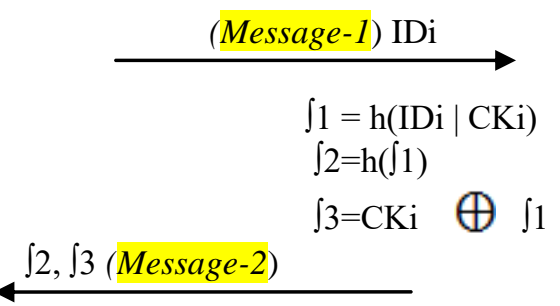

generate Random ${ }_{1}(\mathrm{R} 1 \mathrm{~N})$

$\mathrm{M} 1 \mathrm{~N}=\mathrm{h}\left(\int 2\right) \oplus \mathrm{R} 1 \mathrm{~N}$

$\mathrm{AIDN}=\mathrm{h}(\mathrm{R} 1 \mathrm{~N}) \oplus \mathrm{IDi}$

M2N = h(R1N | M1N | AIDN

(Message-3) M1N,M2N

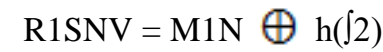

AIDSNV=h(R1SNV) $\oplus \mathrm{IDi}$

M2S $=$ h(R1SNV $\mid$ M1N $\mid$ AIDSNV $)$

check M2S ?= M2N

If $\mathrm{M} 2 \mathrm{~S}==\mathrm{M} 2 \mathrm{~N}$, keep communication generate Random $_{2}(\mathrm{R} 2 \mathrm{~S})$

AIDS=R2S $\oplus \mathrm{h}(\mathrm{IDi})$

$\mathrm{M} 11 \mathrm{~S}=\int 1 \oplus \mathrm{h}(\mathrm{IDi})$

\section{AIDS , M22S (Message-4)}

Blue Eyes Intelligence Engineering

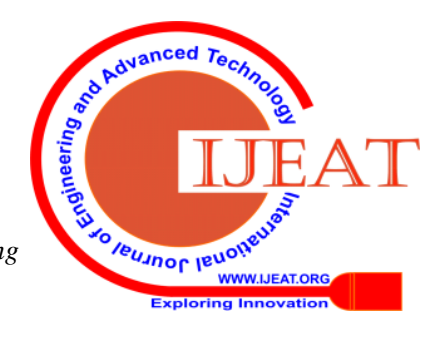




$$
\begin{aligned}
& \text { R2NSV=AIDS } \oplus \mathrm{h}(\mathrm{IDi}) \\
& \int 1=\int 3 \oplus \mathrm{CKi} \\
& \mathrm{M} 11 \mathrm{~N}=\int 1 \oplus \mathrm{h}(\mathrm{IDi}) \\
& \mathrm{M} 22 \mathrm{~N}=\mathrm{h}(\mathrm{M} 11 \mathrm{~N} \mid \text { AIDS | R2NSV }) \\
& \text { If M22S }=\mathrm{M} 22 \mathrm{~N}, \text { keep communication }
\end{aligned}
$$

Figure 1. Delineation of the shared validation stage

Now, shared validation among Node $_{a}$ and server is finished. In this stage, server and Nodea just transmit IDi and check data in cipher content. Secrecy key CKi is just put away locally for decoding, which can diminish the chance of malignant listening stealthily to take keys.

\section{B. Secret Key for Session Synchronization and Data Encryption Phase}

On the off chance that common verification succeeds, both server and Node ${ }_{\mathrm{a}}$ will create a mystery key for meeting and complete the proof that secret key for session generated by both Node $_{\mathrm{a}}$ and server are same by exchanging some encrypted messages. The asset and power utilization of IoT hubs are low, so in our algorithm secret key for session are generated with already existing encrypted messages available on both sides. Along these lines, the heap on the hub and server can be decreased as server concurrently will be communicating with many nodes. The mystery key for meeting synchronization is appeared in Figure 2 and information encryption stage is depicted later.

$$
\text { The Server } \quad \underline{\text { The Node }}
$$

$$
\begin{aligned}
& \text { Generate } \mathrm{SKS}_{1} \\
& \mathrm{SKS}_{1}=\mathrm{h}(\mathrm{R} 1 \mathrm{SNV} \mid \mathrm{R} 2 \mathrm{~S}) \\
& \mathrm{ESKS}_{1}=\mathrm{SKS}_{1} \oplus \mathrm{CKi} \\
& \mathrm{MCKS}=\mathrm{SKS}_{1} \oplus \mathrm{h}(\mathrm{R} 1 \mathrm{SNV})
\end{aligned}
$$

$\stackrel{\mathrm{ESKS}_{1}, \mathrm{MCKS}}{\longrightarrow}$
$\mathrm{SKS}_{1}=\mathrm{ESKS}_{1} \oplus \mathrm{CKi}$
$\mathrm{MCKN}=\mathrm{SKS}_{1} \oplus \mathrm{h}(\mathrm{R} 1 \mathrm{~N})$
Check MCKN ?= MCKS
If MCKN=MCKS , Secret key
received
$\mathrm{CHECKN=MCKN} \oplus \mathrm{h}(\mathrm{R} 2 \mathrm{NSV})$

\section{$\longleftarrow$ CHECKN}

$\mathrm{CHECKS}=\mathrm{MCKS} \oplus \mathrm{h}(\mathrm{R} 2 \mathrm{~N})$

check CHECKS $?=\mathrm{CHECKN}$

If $\mathrm{CHECKS}=\mathrm{CHECKN}$,

Secret key received by node assured

FAS $=h($ R1SNV|R2S $) \oplus$ CHECKS

\section{FAS}

$\mathrm{FAN}=\mathrm{h}(\mathrm{R} 1 \mathrm{~N} \mid \mathrm{R} 2 \mathrm{NSV}) \oplus \mathrm{CHECKN}$

check FAN ? = FAS

If FAN = FAS , Secret key sent by correct

Figure 2. Delineation of the meeting key synchronization stage server assured also synchronization phase ends

Step 1: Server $\rightarrow$ Node $_{a}:\{$ ESKS1,MCKS $\}$. In the wake of affirming the two characters, server produces a mystery key for meeting SKS1. At that point server figures encoded meeting key ESKS1 and check data IV5 as follows:

$$
\begin{aligned}
& \mathrm{SKS}=\mathrm{h}(\mathrm{R} 1 \mathrm{SNV} \mid \mathrm{R} 2 \mathrm{~S}) \\
& \mathrm{ESKS}_{1}=\mathrm{CKi} \oplus \mathrm{SKS}_{1} \\
& \mathrm{MCKS}=\mathrm{SKS}_{1} \oplus \mathrm{h}(\mathrm{R} 1 \mathrm{SNV})
\end{aligned}
$$

From that point onward, server sends $\mathrm{ESKS}_{1}, \mathrm{MCKS}$ to Node $_{\mathrm{a}}$..

Step 2: Node $_{\mathrm{a}} \rightarrow$ Server: $\{\mathrm{CHECKN}\}$. After receiving the message, Node computes $_{\mathrm{a}} \mathrm{SK}_{1}, \mathrm{MCKN}$ and CHECKN by using $\mathrm{CKa}, \mathrm{SKS}_{1}$, Random1 and Random2 as follows:

$$
\begin{aligned}
& \mathrm{SKS}_{1}=\mathrm{ESKS}_{1} \oplus \mathrm{CKi} \\
& \mathrm{MCKN}=\mathrm{SKS}_{1} \oplus \mathrm{h}(\mathrm{R} 1 \mathrm{~N}) \\
& \text { Check MCKN ?= MCKS } \\
& \text { If MCKN=MCKS, } \\
& \text { Secret key received } \\
& \mathrm{CHECKN=MCKN} \oplus \mathrm{h}(\mathrm{R} 2 \mathrm{NSV})
\end{aligned}
$$

If $\mathrm{MCKN}=$ MCKS, Node $_{\mathrm{a}}$ considers mystery key for meeting synchronization is fruitful and then Node $_{a}$ send CHECKN to server so that server gets the assurance that secret key for session is received by Node $_{\mathrm{a}}$ :

Step 3: Server $\rightarrow$ Node $_{\mathrm{a}}:\{$ FAS $\}$ Server receives CHECKN and gets assurance for that Node $_{\mathrm{a}}$ received secret key for

session for synchronization by computing :

$$
\begin{aligned}
& \text { CHECKS = MCKS } \oplus \mathrm{h}(\mathrm{R} 2 \mathrm{~N}) \\
& \text { check CHECKS ? = CHECKN } \\
& \text { If } \mathrm{CHECKS}=\mathrm{CHECKN} \text {, }
\end{aligned}
$$

Secret key received by node assured

FAS $=\mathrm{h}(\mathrm{R} 1 \mathrm{SNV} \mid \mathrm{R} 2 \mathrm{~S}) \oplus \mathrm{CHECKS}$

At this moment, if CHECKS=CHECKN server considers secret key for session is successfully received by Node $\mathrm{a}_{\mathrm{a}}$ and then sends FAS to Node $_{a}$ so that Node $a$ gets a assurance that the data is coming from correct server.

Step 4: Node $_{a}$ receives FAS and checks the data for mystery key for meeting ISKSS with meeting key $\mathrm{SKS}_{1}$ :

$$
\begin{gathered}
\text { FAN }=h(\mathrm{R} 1 \mathrm{~N} \mid \mathrm{R} 2 \mathrm{NSV}) \oplus \mathrm{CHECKN} \\
\text { check FAN ? = FAS }
\end{gathered}
$$

If FAN = FAS , Secret key received is correct and received by correct server is assured. Right now, mystery key for meeting synchronization is finished.

From that point forward, server and Node ${ }_{\mathrm{a}}$ can utilize the present mystery key for session $\mathbf{S K S}_{\mathbf{1}}$ to to generate key for encrypting or decrypting the data for communication.

(i)Encryption Algorithm

Stage 1: Assign all the letter sets for example a to $\mathrm{z}$ with any very much characterized number like 31,22,65,44,23,16, and so forth, it will be spared haphazardly at the two parts of the bargains. For instance

Published By:

Blue Eyes Intelligence Engineering

\& Sciences Publication

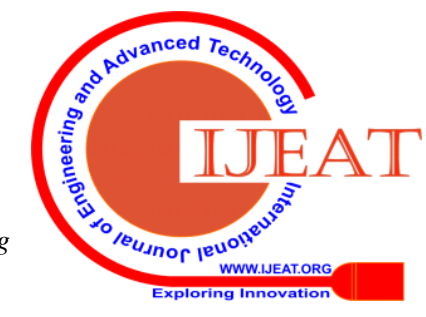


Table III

\begin{tabular}{|c|c|}
\hline Character & Numeric Value \\
\hline $\mathrm{a}$ & 2 \\
\hline $\mathrm{c}$ & 5 \\
\hline $\mathrm{f}$ & 4 \\
\hline $\mathrm{l}$ & 7 \\
\hline $\mathrm{m}$ & 3 \\
\hline $\mathrm{o}$ & 11 \\
\hline $\mathrm{r}$ & 10 \\
\hline $\mathrm{s}$ & 14 \\
\hline $\mathrm{t}$ & 8 \\
\hline $\mathrm{u}$ & 6 \\
\hline $\mathrm{y}$ & 9 \\
\hline $\mathrm{I}$ & 1 \\
\hline
\end{tabular}

Stage 2 In trigonometry activity we require the estimation of $\pi$. At the hour of encryption and unscrambling this estimation of pi will be required. The real estimation of $\pi=3.141592$, however for this situation the Node $_{\mathrm{a}}$ will take the value of $\pi$ be current key generated from the secret key for session $\mathbf{S K N}_{\mathbf{1}}$. For example let the value of key be 1.137201

Here is the method how the key for encryption or decryption is generated from secret key for session(SKSi). First from the secret key for session that is shared, ten different keys be generated ( as secret key for session is defined as int $x[10]$ which is integer array of size 10 and a total of 20 bytes. These 40 bytes are broken into 10 sub keys each of size 2 bytes using the method defined below.

a) First sub key is XOR of all individual element of secret key for session. After this contents of secret key for session is changed. Location zero i.e $x[0]$ now holds the value of location 1 and location 1 holds the value of location 2 and the process continues. Here the last location takes the value of sub key generated above. The actual value of location 0 is discarded. Now the second sub key is created from newly created secret key for session and the above process is repeated till we get all the ten sub keys

b) Once ten sub keys are created, this ten sub keys are reduced to five keys using some simple steps . First key is XOR of first and sixth key followed by left rotation of bits by one. Second key is XOR of second sub key and seventh sub key followed by left rotation of bits by two and the process continues. At the end we are left with five keys.

c) Once we have five keys the next step is XOR of all five keys to get a single key whose size is equivalent to size of int.

d) Finally Modular operation is applied to the output of previous step i.e (output of step-3 ) mod 40 . The output of step- 4 is the final key that will be used for that session until an update takes place.

$$
\text { Shared key=x[10] }=\{2,3,4,5,6,7,8,9,3,1\}
$$

\section{Generated key}

Table IV

\begin{tabular}{|c|c|}
\hline Ki & Value \\
\hline N1 & 2 \\
\hline N2 & 2 \\
\hline N3 & 3 \\
\hline N4 & 4 \\
\hline N5 & 5 \\
\hline N6 & 6 \\
\hline N7 & 7 \\
\hline N8 & 8 \\
\hline N9 & 9 \\
\hline N10 & 3 \\
\hline
\end{tabular}

Final value of Key $=2.00000$

Stage 3: Take the information message . For instance "a bc def" . Here each letters in order will be scrambled by calling the trigonometric capacity $\operatorname{Tan}(\mathrm{x})$, here $\mathrm{x}=\mathrm{a}=1.0$ as haphazardly characterized in sync 1 . For next letters in order $x$ $=\mathrm{b}=2.0$.

These qualities will go into the Tan $(\mathrm{x})$, and gives the cipher content, here Tan (1.0) $=2.1599$, the standard estimation of Tan $(1.0)=1.5574$. As we have changed the estimation of $\pi=\mathrm{SKSi}=1.137201$; the outcome has been naturally changed and giving the figure content.

The general condition of Rule of computing Tan (x) technique is

Tan $\left(\mathrm{x}^{*} \mathrm{pi} / 180.0\right)=\mathrm{y}$ and $\mathrm{CHCK}=\mathrm{CKi} \oplus \mathrm{y}$

Here $\mathrm{y}$ will be considered as cipher content

$$
\begin{aligned}
& \mathrm{y} 1=\operatorname{Tan}\left(\mathrm{x} 1^{*} \mathrm{pi} / 180.0\right) \\
& \text { CHCK1 }=\text { CKi } \oplus \mathrm{y} 1 \\
& \mathrm{y} 2=\operatorname{Tan}(\mathrm{x} 2 * \mathrm{pi} / 180.0) \\
& \text { CHCK }_{2}=\mathrm{CKi} \oplus \mathrm{y} 2 \\
& \mathrm{y} 3=\operatorname{Tan}(x 3 * p i / 180.0)
\end{aligned}
$$$$
\mathrm{CHCK}_{3}=\mathrm{CKi} \oplus \mathrm{y} 3
$$

Presently in the event that $\mathrm{y} 1, \mathrm{y} 2, \mathrm{y} 3$ and encryption calculation are known to the aggressors then additionally they won't have the option to break the data. Since the estimation of isn't general like $\pi=3.141592$ instead of it is 1.137201 (SKS1) and can be relegated with some other worth.

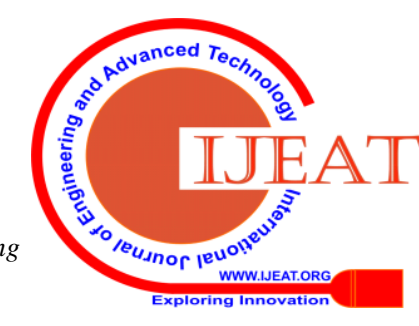


Cryptographic Scheme for Lightweight Device Such as IoT using Pseudo Stream Cipher and Trigonometric Technique with Key Generation

Table V: Demonstration Of Encryption Process

\begin{tabular}{|c|c|c|}
\hline $\begin{array}{c}\text { Plain } \\
\text { Text }\end{array}$ & $\begin{array}{c}\text { Key } \\
\text { (pi) }\end{array}$ & $\begin{array}{c}\text { Tan(x *pi/180.0)/y } \\
\text { CipherText }\end{array}$ \\
\hline I & 2 & 0.989586 \\
\hline space & 2 & 0.937453 \\
\hline a & 2 & 0.999753 \\
\hline m & 2 & 0.999444 \\
\hline space & 2 & 0.937453 \\
\hline f & 2 & 0.999013 \\
\hline a & 2 & 0.999753 \\
\hline c & 2 & 0.998457 \\
\hline u & 2 & 0.997779 \\
\hline l & 2 & 0.996977 \\
\hline t & 2 & 0.996052 \\
\hline y & 2 & 0.995004 \\
\hline space & 2 & 0.937453 \\
\hline f & 2 & 0.999013 \\
\hline r & 2 & 0.993834 \\
\hline o & 2 & 0.99254 \\
\hline m & 2 & 0.999444 \\
\hline space & 2 & 0.937453 \\
\hline k & 2 & 0.991124 \\
\hline i & 2 & 0.999938 \\
\hline t & 2 & 0.996052 \\
\hline s & 2 & 0.987926 \\
\hline
\end{tabular}

(ii) Decryption Process

At the hour of Decryption process , we again require changed estimation of $\pi$. As a figure message the estimation of $\mathrm{y}$ will go into the $\mathrm{aTan}(\mathrm{x})$ work for decoding process.

This is characterized as $\mathrm{x}=\mathrm{aTan}(\mathrm{y}) * 180.0 / \mathrm{pi}$ and CHCK* $=\mathrm{CKi} \oplus \mathrm{x}$ where $\mathrm{x}$ is unique information

$$
\begin{aligned}
& \mathrm{x} 1=\mathrm{aTan}(\mathrm{y} 1)^{*} \mathrm{pi} / 180.0--(1) \\
& \mathrm{CHCK} 1 *=\mathrm{CKi} \oplus \mathrm{x} 1
\end{aligned}
$$

Just if CHCK1 ${ }^{*}=\mathrm{CHCK}^{*}$, the receiver(server) will keep correspondence. Something else, collector (server) feels that the information has been malevolent altered. CHK is utilized to secure information trustworthiness. In the event that $\mathrm{CHCK}^{*}=\mathrm{CHCK}^{*}$, here in any case key won't be split by the unapproved client. It takes care of the issue of Cryptanalysis.

Here key is $\pi$. The estimation of $\pi$ (going about as key) is known to approve client as it were.

Table VI: Demonstration Of Decryption Process

\begin{tabular}{|c|c|c|c|}
\hline Ciphertext & Key(pi) & $\begin{array}{c}\text { aTan(y)*180/p } \\
\mathbf{~ i}\end{array}$ & $\begin{array}{c}\text { Plaintext } \\
\mathbf{X}\end{array}$ \\
\hline 0.989586 & 2 & 13 & $\mathrm{I}$ \\
\hline 0.937453 & 2 & 32 & space \\
\hline 0.999753 & 2 & 2 & $\mathrm{a}$ \\
\hline 0.999444 & 2 & 3 & $\mathrm{~m}$ \\
\hline 0.937453 & 2 & 32 & space \\
\hline 0.999013 & 2 & 4 & $\mathrm{f}$ \\
\hline 0.999753 & 2 & 2 & $\mathrm{a}$ \\
\hline 0.998457 & 2 & 5 & $\mathrm{c}$ \\
\hline 0.997779 & 2 & 6 & $\mathrm{u}$ \\
\hline
\end{tabular}

\begin{tabular}{|c|c|c|c|}
\hline 0.996977 & 2 & 7 & $\mathrm{l}$ \\
\hline 0.996052 & 2 & 8 & $\mathrm{t}$ \\
\hline 0.995004 & 2 & 9 & $\mathrm{y}$ \\
\hline 0.937453 & 2 & 32 & space \\
\hline 0.999013 & 2 & 4 & $\mathrm{f}$ \\
\hline 0.993834 & 2 & 10 & $\mathrm{r}$ \\
\hline 0.99254 & 2 & 11 & $\mathrm{o}$ \\
\hline 0.999444 & 2 & 3 & $\mathrm{~m}$ \\
\hline 0.937453 & 2 & 32 & $\mathrm{space}$ \\
\hline 0.991124 & 2 & 12 & $\mathrm{k}$ \\
\hline 0.999938 & 2 & 1 & $\mathrm{i}$ \\
\hline 0.996052 & 2 & 8 & $\mathrm{t}$ \\
\hline 0.987926 & 2 & 14 & $\mathrm{~s}$ \\
\hline
\end{tabular}

(iii) Pseudo Stream Cipher:

In this stage, we put forth another figure component called pseudo stream figure. Standard stream figure is snappy and gainful yet it encounters an issue of key exchange. In pseudo stream figure, there is no convincing motivation to guarantee synchronization of key age. It gives a framework that the key can be delivered by a side of the correspondence, for instance, the server. By then the key is synchronized through the encryption of an unpredictable and check for the figure content.

Pseudo stream figure improves key synchronization and decreases the overhead of IoT center points.

\section{MYSTERY KEY FOR UPCOMING SESSION}

In order to improve the security of correspondence, the arrangement uses a variable gathering key. The gathering key update period and key length can be adjusted by different applications. In case the government operative needs vindictive listening covertly, the gathering key must be broken consistently which is irksome as encryption and unraveling keys are made from riddle key for meeting.

Exactly when the normal data correspondence time reciprocals to a gathering key update period, server produces information for update puzzle key for immediately (new) meeting (IUSKS) and encodes it:

\section{EIUSKS $=\mathrm{CKa} \oplus \operatorname{IUSKS}(12)$}

By then server sends EIUSKS(encrypted information for update secret key for next(new) meeting) to the Node ${ }_{\mathrm{a}}$. In the wake of getting this information, center stops sending data and holds on for another gathering key. Starting now and into the foreseeable future, server makes another gathering key (for instance second gathering so SKS2). The following system is equal to the gathering key synchronization process.

Published By:

Blue Eyes Intelligence Engineering \& Sciences Publication

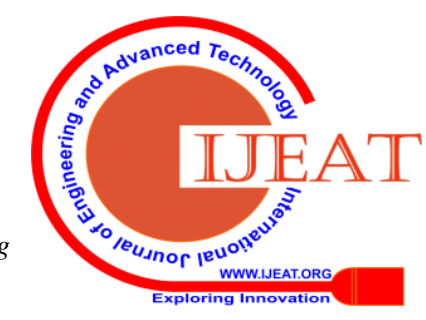


The whole of the above are the nuances of the lightweight arrangement. The arrangement fuses three phases. In like manner approval stage, we present a mystery key CKa and masterminded a solitary course hash work. It can comprehend regular confirmation just as hinder various attacks. In both Mutual Authentication and key synchronization stage, pseudo stream figure was proposed to smooth out approval and key synchronization. Variable gathering key is sensible for different application and hinders ambushes satisfactorily. It furthermore has an orchestrated key age limits at both the ends(Hub and server)

\section{SECURITY ANALYSIS}

In this Section, we give the security examination of the proposed confirmation instrument. We have received the security investigation approach followed in and in this way, we have the accompanying:

Recommendation 1: Key namelessness is given by the proposed system.

Verification: The keys in our arrangement are never transmitted in plain substance, they are continually transmitted in figure message, paying little heed to the mystery key CKi or meeting key SKSi.Additionally from this secret key for session key for encryption and decoding are created . Additionally, the gathering key SKSi is invigorating. This can thwart the spillage of keys to noxious aggressors.

Suggestion 2: The proposed component gives substance shared confirmation.

Verification: In the confirmation stage, common validation between the Node $_{\mathrm{a}}$ and the server can be accomplished dependent on the got Message 3 and Message 4. Upon receipt of M1N and M2N, the switch checks whether M2N is equivalent to $\mathrm{h}(\mathrm{R} 1 \mathrm{SNV}|\mathrm{M} 1 \mathrm{~N}| \mathrm{AIDSNV})$ ). The savvy sensor is viewed as verified if the fairness holds. A similar procedure happens in validating the server when the brilliant sensor gets AIDN and M22N . The brilliant sensor processes h(M11N | AIDS | R2NSV) and checks whether this worth is equivalent to M22N . On the off chance that they are equivalent, the switch is additionally considered as verified. Additionally, if the foe expects to fashion a substantial Node / $_{\text {switch, he/she }}$ needs to create legitimate messages. Notwithstanding, the foe can't create the substantial messages since he has no data about the irregular numbers(i.e.,Random1 and Random2).

Recommendation 3: Mystery key for Session synchronization and invigorating.

Affirmation: The arrangement can uses a variable gathering key to improve the security of correspondence. As demonstrated by different applications, standard for key invigorating varies. For tricky or high-traffic data, we can manufacture the update repeat and the key length. In any case, if the data is coldhearted or low-traffic, the key update repeat and the key length can be diminished.

Suggestion 4: The proposed instrument is impervious to replay assault.

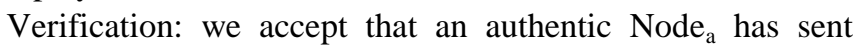
Message 3 (i.e., M1N and M2N ) to the server. On the off chance that an enemy attempts to mimic the genuine Node $\mathrm{a}_{\mathrm{a}}$ by replaying Message 3, the server will dismiss the confirmation demand in light of the fact that the M2N of the shrewd sensor is determined dependent on a hash estimation of an arbitrary number Random1 which is just known to the real Node . $_{\text {. }}$

Suggestion 5: The proposed system is impervious to man-in-the-middle attack.

Proof: This arrangement can reasonably thwart man-in-the-inside attacks because of strong approval of character, understanding for different letters all together in encryption and unscrambling limit and strong decency on the data. Any alteration on the characteristics in like manner confirmation stage will cause the missing the mark checking of M2N/M2S or M22N/M22S. Also, paying little heed to the server and the center point will check the data uprightness by checking the CHCK data. If the data is changed, the server or the center will excuse this correspondence.

Recommendation 6: The proposed component is impervious to pantomime assault.

Confirmation: To adequately complete a mask ambush, the aggressor must pass check in the mutual approval stage and to have the alternative to interpret the check message precisely. The security key CKi of Nodea is never moved in plaintext in the approval stage. Furthermore, it's hard for attacker to get the arranged key age work and masterminded one way hash work. Over all how all the letter sets are deciphered in Encryption and Decryption also the last check by CHCK makes it the most irksome task for attacker.

Suggestion 7: The proposed instrument is impervious to alteration assault.

Evidence: The single direction hash work $h(\cdot)$ ensures that data can't be altered without being distinguished. On the off chance that a foe transmits an altered message to the switch, the switch will identify it by checking the hash esteems.

Security properties of the proposed plot, differentiated and related works are summed up in Table VII.

Table VII Security examination of our plan and related

\begin{tabular}{|c|c|c|c|c|}
\hline Items & $\begin{array}{c}\text { Our } \\
\text { Schem } \\
\text { e }\end{array}$ & SASI & $\begin{array}{c}\text { Goassamer } \\
\text { protocol }\end{array}$ & $\begin{array}{c}\text { Dynamic Key } \\
\text { Scheme-Yuxin \& } \\
\text { Wang(2018) }\end{array}$ \\
\hline $\begin{array}{c}\text { Mutual } \\
\text { authentica } \\
\text { tion }\end{array}$ & $\sqrt{ }$ & $\sqrt{ }$ & $\sqrt{ }$ & $\sqrt{ }$ \\
\hline $\begin{array}{c}\text { Key } \\
\text { secrecy }\end{array}$ & $\sqrt{ }$ & $\sqrt{ }$ & $\sqrt{ }$ & $\sqrt{ }$ \\
\hline $\begin{array}{c}\text { Meeting } \\
\text { key } \\
\text { synchroni } \\
\text { zation }\end{array}$ & $\sqrt{ }$ & $\times$ & $\times$ & $\sqrt{ }$ \\
\hline $\begin{array}{c}\text { Protection } \\
\text { to } \\
\text { impersona } \\
\text { tion attack }\end{array}$ & $\sqrt{ }$ & $\times$ & $\sqrt{ }$ \\
\hline $\begin{array}{c}\text { Protection } \\
\text { from } \\
\text { replay } \\
\text { assult }\end{array}$ & $\sqrt{ }$ & $\times$ & $\times$ & $\sqrt{ }$ \\
\hline $\begin{array}{c}\text { Protection } \\
\text { from } \\
\text { man-in-ce } \\
\text { nter } \\
\text { assault }\end{array}$ & $\sqrt{ }$ & $\times$ & $\times$ & $\sqrt{ }$ \\
\hline
\end{tabular}

Published By:

Blue Eyes Intelligence Engineering \& Sciences Publication

(C) Copyright: All rights reserved. 


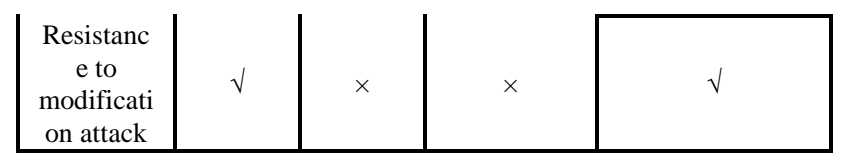

When our scheme is compared with dynamic key scheme by Yuxin and Wang(2018)[12] dynamic key security provides only one level security i.e.. with key (key is passed secretly , key size can be changed and key is updated periodically) whereas in our scheme key apart from above security level, three more security features are added .Each character is assigned a number which is hidden from outsider, second by using the secret key which is shared a key whose size is equivalent to the size of integer is generated, this key is used for encryption and decryption and at the end the data which is encrypted is checked at the receiving end so when we compare our scheme with dynamic key scheme three extra level of security is introduced in our scheme

\section{PERFORMANCE ANALYSIS}

We consider the hour of the mutual affirmation stage, which is completed [15][16] in the four shows (SASI, Our arrangement, dynamic key and Gossamer show). In this investigation, the plans are coordinated on a work regions with windows 32bit, CPU $2.00 \mathrm{GHz}$ with $2 \mathrm{~GB}$ of RAM. Exploratory outcomes are the ordinary time of three investigation for four shows (furthermore the results are appeared and the test are done contemplating two marks/IoT contraption are annexed to server notwithstanding in every one of the three cases 20 bytes are considered for keys in four cases) and showed up in Figure 3. In the wake of analyzing Figure 3, we notice that our arrangement achieves the best time execution of 15500 ms, Dynamic key has 16000ms , SASI moreover has incredible execution of $31000 \mathrm{~ms}$ and Goassamer show has the greatest time

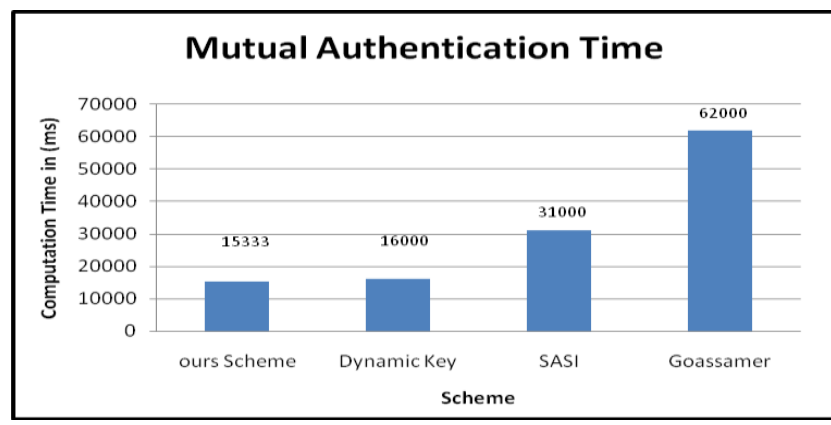

Figure 3. Correlation of shared confirmation time among

SASI, Our plan and Goassamer convention and dynamic key plan Wang\&Yuxin(2018)

Our proposed plot is less overhead, and can oppose different assaults. Dynamic key uses XOR and Hash work, give shared verification and impervious to the greater part of the assault however when contrasted and our plan the quantity of security level are less . SASI uses bitwise XOR, extension modulo $2 \mathrm{~m}$ and revolution to recognize normal affirmation. They cost low usages yet give obliged security features. Goassamer uses bitwise XOR, expansion modulo $2 \mathrm{~m}$, transformation and mixbits ability to recognize shared approval. Differentiated and the Dynamic Key, SASI and Goassamer, our arrangement takes less overhead and gives more prominent security features. In our arrangement, we simply use XOR action , one way Hash work or possibly action for shared approval and meeting key synchronization. Additionally, secret key is made by server and synchronized with IoT center point. It is a better than average technique to diminish the overhead of IoT center points.

The examination of the proposed trigonometry computation for encryption and translating has been done and appeared in Figure2 and Figure3. The Encryption and Decryption Algorithm was coded in C Language[15][16]. It was aggregated with MinGW-GCC 4.8.1, on the Core 2 Duo Processor, $2.00 \mathrm{GHz}$ under windows 7 OS. The assessment parameters are plain substance size (in Bytes) and time taken in encryption and disentangling (in microseconds).

Table VIII: Execution Times For Encryption Algorithm On Core(TM) 2 Duo, 2.00 GHZ

\begin{tabular}{|c|c|}
\hline $\begin{array}{c}\text { Size(In } \\
\text { Byte) }\end{array}$ & $\begin{array}{c}\text { Time } \\
(\mu \mathrm{sec})\end{array}$ \\
\hline 8 & 62000 \\
\hline 19 & 109000 \\
\hline 27 & 141000 \\
\hline 35 & 156000 \\
\hline 42 & 203000 \\
\hline
\end{tabular}

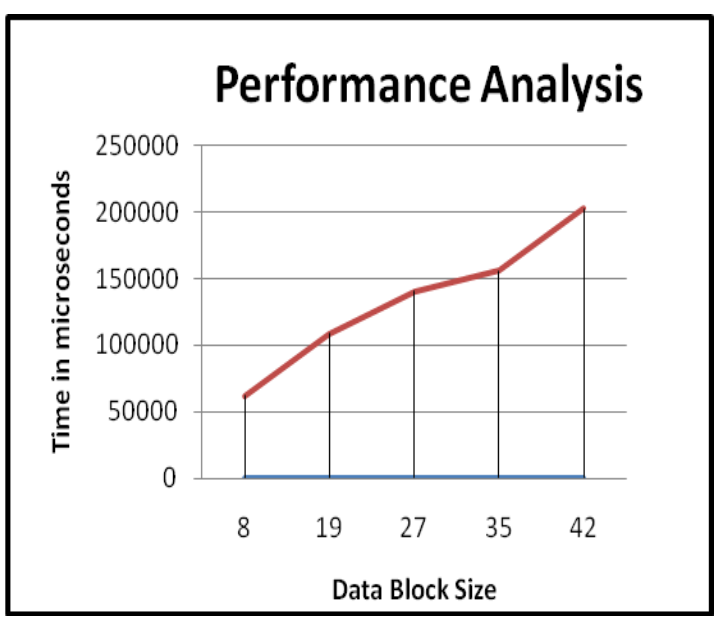

Fig. 4: Performance analysis of encryption

Table IX: Execution Times For Decryption Algorithm On Core(TM) 2 Duo, 2.00 GHZ.

\begin{tabular}{|c|c|}
\hline $\begin{array}{c}\text { Size(In } \\
\text { Byte) }\end{array}$ & $\begin{array}{c}\text { Time } \\
(\mu \mathrm{sec})\end{array}$ \\
\hline 8 & 0 \\
\hline 16 & 15500 \\
\hline 24 & 31000 \\
\hline 32 & 47000 \\
\hline 40 & 63000 \\
\hline
\end{tabular}




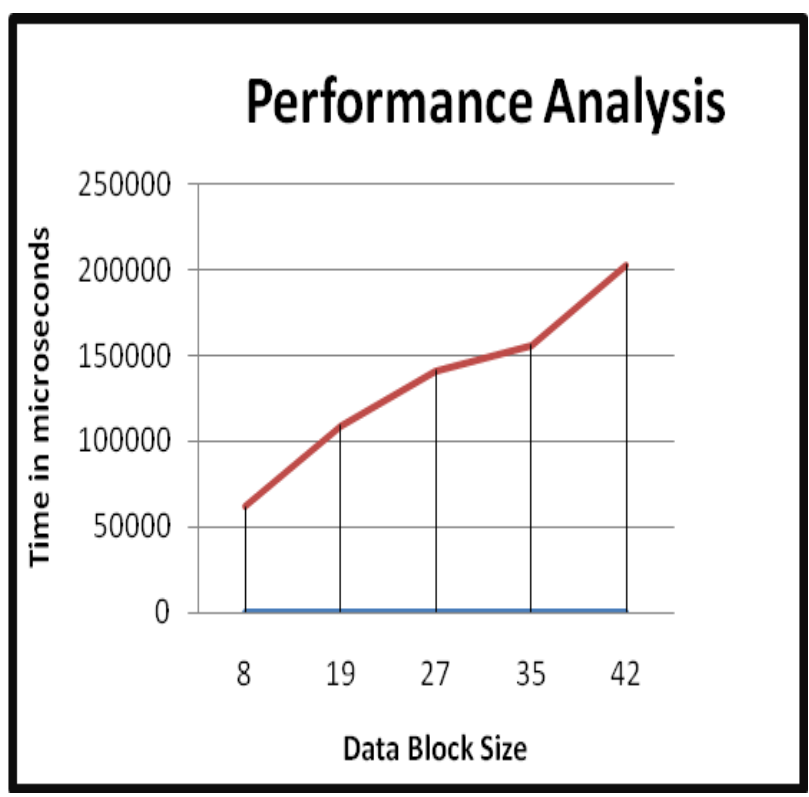

Fig. 5: Performance analysis of Decryption

\section{KNOWN PLAIN TEXT CRYPTANALYSIS ATTACK CHECKING ON TRIGNOMETRIC ALGORITHM}

Assume here that for any plaintext the assailant has a figure content which is "0.527718 0.6732190 .642737 ", now how about we check the assaulter will have the option to break the plain content or not , and we expect that the aggressor is notable about the calculation. From the outset aggressor accept that the plain content is $\mathrm{x} 1 \mathrm{x} 2$ x3 and all these are factors and those might be a genuine number . Furthermore, the estimation of $\pi 1$ isn't known to him

$$
\begin{gathered}
0.527718=\operatorname{Tan}(x 1 * \pi / 180.0) \\
0.673219=\operatorname{Tan}(x 2 * \pi / 180.0)
\end{gathered}
$$

$$
0.642737=\operatorname{Tan}(x 3 * \pi / 180.0
$$

From equation (i)

$$
\begin{aligned}
& \operatorname{Tan}^{-1}(0.527718)=x 1 * \pi / 180.0 \\
& \pi=5007.855148 / x 1
\end{aligned}
$$

From condition (ii)

$$
\begin{aligned}
& \operatorname{Tan}^{-1}(0.673219)=x 2 * \pi / 180.0 \\
& \pi=6110.8540 / x 2
\end{aligned}
$$

From condition (iii)

$$
\begin{aligned}
& \operatorname{Tan}^{-1}(0.642737)=x 3 * \pi / 180.0 \\
& \pi=5891.4639 / \times 3
\end{aligned}
$$

From the above equation we can get that

$\pi=5007.855148 / x 1=6110.8540 / x 2=5891.4639 / x 3$

So now from the above condition the assailant won't have the option to get the estimation of $\mathrm{x} 1, \mathrm{x} 2, \mathrm{x} 3$. Since the estimation of $\pi$ is diverse as opposed to the customary worth. So it is demonstrated that the tomb examination assault is neglected to break the trigonometric cipher.

\section{COMPARATIVE PERFORMANCE ANALYSIS}

The proposed figuring has been differentiated and other existed calculations like RC4, Hill-Cipher[17][18], RSA ,

\begin{tabular}{|c|c|c|c|c|c|c|c|}
\hline \multicolumn{8}{|c|}{ Time in $\mu$ sec for different Algorithms } \\
\hline \multirow[b]{2}{*}{$\begin{array}{l}\text { No of } \\
\text { Bytes }\end{array}$} & RC4 & \multirow[b]{2}{*}{$\begin{array}{c}\text { Hill-Cipher } \\
\text { (Symetric } \\
\text { encryption) }\end{array}$} & RSA & Trigonometry & Present & ELSCA & COZMO \\
\hline & $\begin{array}{l}\text { (strea } \\
\text { m } \\
\text { cipher } \\
\text { ) }\end{array}$ & & $\begin{array}{l}\text { (public key } \\
\text { encryption) }\end{array}$ & $\begin{array}{c}\text { (LW-psudo } \\
\text { stream } \\
\text { cipher) }\end{array}$ & $\begin{array}{c}\text { (LW-Block } \\
\text { Cipher (64 } \\
\text { bits)) }\end{array}$ & $\begin{array}{c}\text { (LW-StreamCiph } \\
\text { er-2017) }\end{array}$ & $\begin{array}{c}\text { (LW-StreamCiph } \\
\text { er-2018) }\end{array}$ \\
\hline 8 & 0 & 0 & 16000 & 0 & 16000 & 0 & 0 \\
\hline 16 & 15000 & 32000 & 31000 & 15500 & 32000 & 16000 & 0 \\
\hline 24 & 31000 & 63000 & 46000 & 31000 & 48000 & 62000 & 31000 \\
\hline 32 & 31000 & 78000 & 62000 & 47000 & 64000 & 62000 & 47000 \\
\hline 40 & 62000 & 63000 & 78000 & 63000 & 80000 & 93000 & 62000 \\
\hline
\end{tabular}
Present(Block Cipher with key 80 bits and plain content 64 bits or bytes)[13], COZMO (LW-StreamCipher-2018)[[20][21][22][23][24]andELSCA( LW-StreamCipher-2017)[25][26][27].

Likewise, the results have showed up in the going with chart. From the going with figure we can say that the run time unpredictability of trigonometric Algorithm is underneath than the other existed Algorithm plot.
Published By:

Blue Eyes Intelligence Engineering \& Sciences Publication

(C) Copyright: All rights reserved.

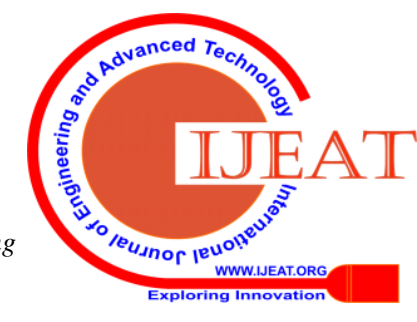




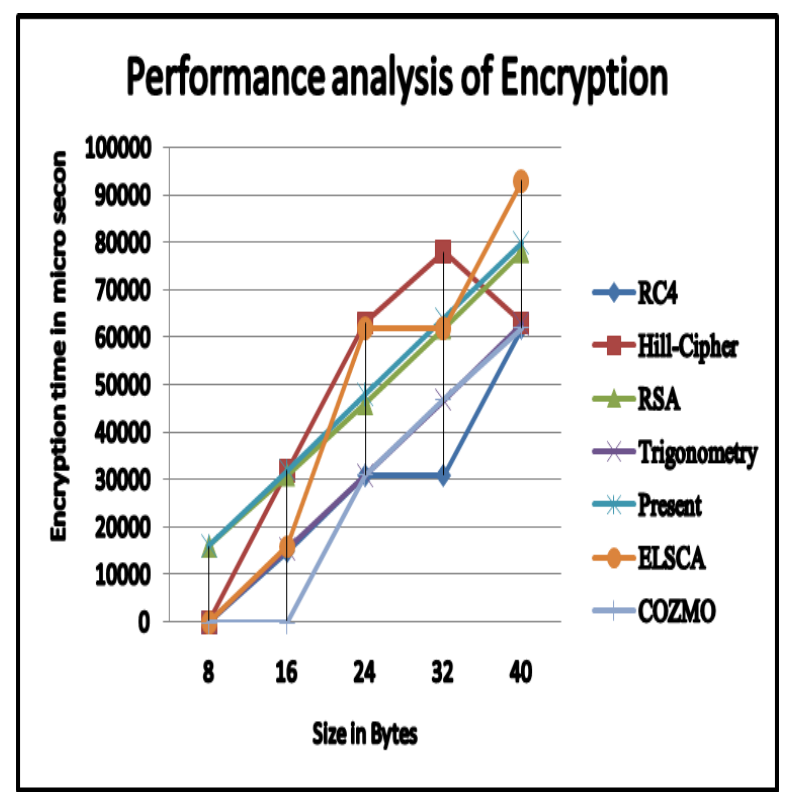

Fig 6: Performance analysis of Encryption

\section{CONCLUSION}

In this paper, we propose a lightweight cryptographic count and endeavored to improved security and execution of a cryptographic arrangement for lightweight contraption using key age and pseudo stream figure.

It gives monetarily sagacious and possible instruments for basic affirmation, meeting key synchronization and meeting key update. They are proper for IoT center point with obliged enlisting resources and force. Directly off the bat, we present the nuances of normal approval using pseudo stream figure and a way to deal with prevent replay attack without timestamp. Moreover, again pseudo stream figure is progressed to simply recognize meeting key synchronization lastly by using trigonometric thoughts data encryption and unscrambling are done to reduce the overhead of IoT center points. In addition, we have taken a gander at the security and execution of our arrangement with some lightweight check and cryptographic plans. The arrangement is lightweight anyway can hinder attacks effectively, which is fit for guaranteeing the security of the correspondence between IoT center points and servers.

The proposed calculation for encryption isn't simply giving the fast data encryption yet it gives a prevalent security appeared differently in relation to other computation through a most grounded key also. The estimation of $\pi$ is changing each time which is delivered from the regular key that makes the computation adequate. The count furthermore makes the cryptanalysis methodology complex. Since there are dark components will be more and the amount of conditions will be less when appeared differently in relation to other standard encryption figurings. This estimation could be well utilitarian for online applications like visiting. The assessment of Encryption layout reflects the data square size and encryption time as a straight association, after the square size of 32 Bytes.

\section{REFERENCES}

1. J. Gubbi, R. Buyya, S. Marusic, and M. Palaniswami, "Internet of things (iot): A vision, architectural elements, and future directions,"
FutureGeneration Computer Systems, vol. 29, no. 7, pp. 1645-1660, 2013.

2. Lin J, Yu W, Zhang N, Yang X, Zhang H and Zhao W, "A Survey on Internet of Things: Architecture, Enabling Technologies, Security and Privacy, and Applications", IEEE Internet of Things Journal, 2017, PP(99):1-1.

3. Mahmoud R, Yousuf T, Aloul F and Zualkernan I, "Internet of things (IoT) security: Current status, challenges and prospective measures", Internet Technology and Secured Transactions, IEEE, 2016:336-341.

4. S. A. Kumar, T. Vealey, and H. Srivastava, "Security in internetof things: Challenges, solutions and future directions," in $201649^{\text {th }}$ Hawaii International Conference on System Sciences (HICSS). IEEE, 2016, pp. 5772-5781.

5. M. Katagi and S. Moriai, "Lightweight cryptography for the internet of things," Sony Corporation, pp. 7-10, 2008.

6. M. Ebrahim, S. Khan, and U. B. Khalid, "Symmetric algorithm survey:A comparative analysis," International Journal of Computer Applications(0975 - 8887), vol. 61, no. 20, 2014.

7. Chien H Y. SASI: A New Ultralightweight RFID Authentication Protocol Providing Strong Authentication and Strong Integrity[M]. IEEE Computer Society Press, 2007.

8. CAO T, BERTINO E, LEI H. Security Analysis of the SASI Protocol[J]. IEEE Transactions on Dependable and Secure Computing. 2009, 6(1), pp. 73-77.

9. Peris-Lopez P, Hernandez-Castro J C, Tapiador J M E, et al. Advances in Ultralightweight Cryptography for Low-Cost RFID Tags: Gossame Protocol[C]// Information Security Applications. Springer- Verlag, 2009, pp. 56-68

10. TIAN Y, CHEN G L, LI J. A New Ultra-lightweight RFID Authentication Protocol with Permutation[J]. IEEE Communications Letters. 2012, 16(5), pp. 702-705.

11. WANG S H, HAN Z J, LIU S J, et al. Security Analysis of RAPP: an RFID Authentication Protocol Based on Permutation[R]. Cryptology ePrint Archive, Report 2012/327, 2012.

12. Yuxin Wu , Jing Wang “A Light weight Cryptographic Scheme with Dynamic Key" $201810^{\text {th }}$ International Conference on Communication Software and Network

13. A. Bogdanov, L. R. Knudsen, G. Leander, C. Paar, A. Poschmann, M. J.Robshaw, Y. Seurin, and C. Vikkelsoe, "Present: An ultra-lightweight block cipher," in International Workshop on Cryptographic Hardware and Embedded Systems. Springer, 2007, pp. 450-466.

14. B. Ray, S. Douglas, S. Jason, T. Stefan, W. Bryan, and W. Louis, "The simon and speck families of lightweight block ciphers," Cryptology ePrint Archive, Report./404, Tech. Rep., 2013.

15. 3GPP TS 35. 201 V14. 0. 0, Specificatio n of the 3GPP Confidentiality and Integrity Algorithms; Document 1: f8 and f9 specifications, 2017.

16. 3GPP TS 35. 202 V14. 0. 0, Specificatio n of the 3GPP Confidentiality and Integrity Algorithms; Document 2: Kasumialgorithm specifications, 2017.

17. Ghada Hamissa, Hatem Abdelkader, "Securing JPEG Architecture Based on Enhanced Chaotic Hill Cipher Algorithm" ,IEEE2011.

18. Ahmed Desokyi, Anju Panicker Madhusoodhanan, "Bitwise Hill Crypto System", IEEE 2011.

19. Vimalathithan.R , Dr.M.L.Valarmathi, "Cryptanalysis of S- DES using Genetic Algorithm", International Journal of Recent Trends in Engineering, November 2009.

20. Rhea Bonnerji, Simanta Sarkar, Krishnendu Rarhi, Abhishek Bhattacharya "COZMO - A New Lightweight Stream Cipher" 978-1-5386-5657-0/18/\$31.00_c 2018 IEEE

21. B. Preneel, "Trivium - A Stream Cipher Construction Inspired by Block Cipher Design Principles," (2006)

22. Andy Rupp,“A Real-World Attack Breaking A5/1 within Hours, (2008).

23. Raddum, H. “Cryptanalytic Results on Trivium”, eSTREAM, 2006.

24. Biham E., Cryptanalysis of the A5/1 GSM stream cipher. Springer, 2000.

25. Soumyadev Maity, Koushik Sinha, Bhabani P. Sinha "An Efficient Lightweight Stream Cipher Algorithm for Wireless Networks" 978-1-5090-4183-1/17/\$31.00 (2017 IEEE

26. S. S. Gupta, A. Chattopadhyay, K. Sinha, S. Maitra and B. P. Sinha, "High performance hardware implementation for RC4 stream cipher," IEEE Trans. on Computers, vol. 62(4), April 2013, pp. 730-743.

Published By:

Blue Eyes Intelligence Engineering

\& Sciences Publication

(C) Copyright: All rights reserved.

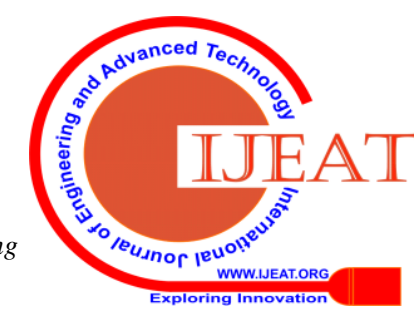


27. M. B. Shemaili, C. Y. Yeun, K. Mubarak and M. J. Zemerly, "A new lightweight hybrid cryptographic algorithm for the internet of things," Intl. Conf. for Internet Technology and Secured Transactions, London, 2012, pp. 87-92

\section{AUTHORS PROFILE}

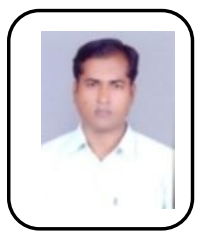

Bhaskar Prakash Kosta received his MCA(NIT Calicut, India 1998) and ME CSE (Anna University, India 2007). He is currently is pursuing a $\mathrm{PhD}$ in Computer Science and Engineering. He has about 15 years of experience in Teaching. His research interests lie in computer network, databases ,network and information security and in the design and implementation of cryptographic algorithm for various devices

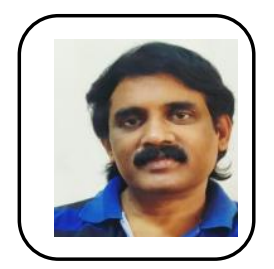

Dr. P Sanyasi Naidu His research area includes Applied Cryptography, Network and Cloud Security. He got prestigious "Governor's National Award for Excellence in Research and Development" with full honors and privileges in recognition of his remarkable achievements and outstanding contribution in the field of Research, Publications, Patents, Training, Mentoring, \& Teaching in engineering discipline. He is one of the top performer of the certification courses conducted by IIT Bombay "Foundation Program in ICT for Education", "Pedagogy for Online and Blended Teaching-Learning Process", "Mentoring Educators in Education Technology". He got 10 ELITE NPTEL certificates conducted by various IIT's for successful completion and Mentoring of various advanced Computer Engineering Courses and Mezzanine Technologies

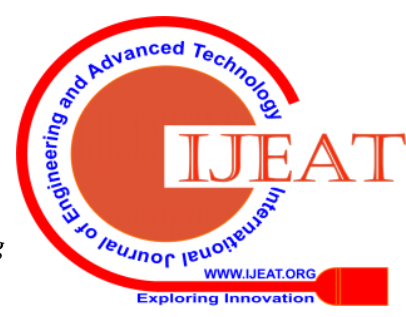

